\title{
Article
}

\section{Power System Reconfiguration in Distribution Network for Improving Reliability Using Genetic Algorithm and Particle Swarm Optimization}

\author{
Omar Kahouli 1,2,*, Haitham Alsaif ${ }^{3}$, Yassine Bouteraa ${ }^{4}{ }^{\oplus}$, Naim Ben Ali ${ }^{5,6}$ and Mohamed Chaabene ${ }^{7}$ \\ 1 Department of Electronics Engineering, Community College, University of Ha'il, Ha'il 81481, Saudi Arabia \\ 2 Control \& Energies Management (CEM-Lab), National Engineering School of Sfax, University of Sfax, \\ Sfax 3038, Tunisia \\ 3 Department of Electrical Engineering, College of Engineering, University of Ha'il, Ha'il 2440, Saudi Arabia; \\ h.alsaif@uoh.edu.sa \\ 4 Department of Computer Engineering, College of Computer Engineering and sciences, Prince Sattam bin \\ Abdulaziz University, Al-Kharj 11942, Saudi Arabia; y.bouteraa@psau.edu.sa \\ 5 Department of Industrial Engineering, College of Engineering, University of Ha'il, Ha'il 2440, Saudi Arabia; \\ na.benali@uoh.edu.sa \\ 6 Photovoltaic and Semiconductor Materials Laboratory, National Engineering School of Tunis, University of \\ Tunis El Manar, Tunis 1002, Tunisia \\ 7 Laboratory of Sciences and Techniques of Automatic Control \&Computer Engineering, National Engineering \\ School of Sfax, University of Sfax, Sfax 3038, Tunisia; mohamed.chaabane@enis.tn \\ * Correspondence: a.kahouli@uoh.edu.sa
}

Citation: Kahouli, O.; Alsaif, H.; Bouteraa, Y.; Ben Ali, N.; Chaabene, M. Power System Reconfiguration in Distribution Network for Improving Reliability Using Genetic Algorithm and Particle Swarm Optimization. Appl. Sci. 2021, 11, 3092. https:// doi.org/10.3390/app11073092

Academic Editor: Eunsung Oh

Received: 22 February 2021

Accepted: 24 March 2021

Published: 31 March 2021

Publisher's Note: MDPI stays neutral with regard to jurisdictional claims in published maps and institutional affiliations.

Copyright: (c) 2021 by the authors. Licensee MDPI, Basel, Switzerland. This article is an open access article distributed under the terms and conditions of the Creative Commons Attribution (CC BY) license (https:// creativecommons.org/licenses/by/ $4.0 /)$.

\begin{abstract}
This paper presents an optimal method for optimizing network reconfiguration problems in a power distribution system in order to enhance reliability and reduce power losses. Network reconfiguration can be viewed as an optimization problem involving a set of criteria that must be reduced when adhering to various constraints. The energy not supplied (ENS) during permanent network faults and active power losses are the objective functions that are optimized in this study during the reconfiguration phase. These objectives are expressed mathematically and will be integrated into various optimization algorithms used throughout the study. To begin, a mathematical formulation of the objectives to be optimized, as well as all the constraints that must be met, is proposed. Then, to solve this difficult combinatorial problem, we use the exhaustive approach, genetic algorithm (GA), and particle swarm optimization (PSO) on an IEEE 33-bus electrical distribution network. Finally, a performance evaluation of the proposed approaches is developed. The results show that optimizing the distribution network topology using the PSO approach contributed significantly to improving the reliability, node voltage, line currents, and calculation time.
\end{abstract}

Keywords: electrical distribution network; reconfiguration problem; reliability; energy not supplied; active power losses; exhaustive research; genetic algorithm; particle swarm optimization

\section{Introduction}

Electrical distribution networks are a critical component of the electrical power system. They are generally denser and more complex than the transportation networks that supply them through transformer stations [1]. Given the ever-increasing complexity of distribution networks, automation of previously manual tasks is becoming increasingly more important. To that end, new tools that aid in the operation of these networks, known as advanced automation functions in the literature, have emerged. The role of these functions is to provide the distribution network operator with the ability to solve network problems as they occur [2]. Furthermore, in order to ensure a secure economically optimal electricity supply, it is important to use distribution network reconfiguration to find the best solutions based on the requirements and constraints defined by the operators. 
Distribution network reconfiguration can be defined as the process of altering the topological structures of the distribution network by changing the open/close status of the sectionalizing and tie switches. This process can improve the performance of the system according to different particular objectives and constraints [3].

In recent years, applied researchers have become increasingly interested in finding the optimal distribution network reconfiguration taking into account several objectives. A close literature review indicates that reliability enhancement has always been one of the main objectives [4]. Besides, much attention was focused on other criteria such as active power loss minimization, load balancing. and voltage stability security margin $[5,6]$.

The reliability of an electrical network is described as the ability of the power system to supply consumers uninterruptedly with an adequate quality electricity supply. Some of the methods for reliability enhancement are as follows: adding new devices for protection, utilization of more reliable equipment to avoid contingencies, fast switching and reclosing schemes, accurate precise fault allocation techniques, fast crew to speed up the repair process, and system topology reconfiguration [7].

The reliability of distribution networks has become increasingly important in the deregulated power sector [8,9]. To address these issues, distribution network reconfiguration is regarded as a cost-effective and efficient way to increase system reliability. The reliability index considered to measure the reliability of the distribution network in this work is the energy not supplied (ENS). This index forecasts the amount of energy that will be lost due to failures over a given time period. Based on the accurate estimation of the ENS index, appropriate planning and design initiatives in distribution systems can be implemented to provide consumers with cost-effective and reliable power.

In addition to reliability improvement, active power loss reduction of a power system enhances the delivery of better power quality [10]. There are many measures to reduce active power losses inthe distribution system such as operating at higher voltage levels, installing a distribution generator or capacitor, and network reconfiguration. specifically, network reconfiguration is an effective measure to reduce active power losses because it does not require any additional cost. The objective functions for the network reconfiguration considered in this work are the active power losses and ENS reliability index.

The distribution network topology can be altered by performing the action of opening/closing on the sectionalizing and tie switches. Since the number of switches isrelatively high in a large distribution network, it is almost impossible for the operator to find the best topology without an optimization study. The distribution network reconfiguration problem was first solved by [11]. Since then, many algorithms have been developed for reliability improvement and power loss reduction in distribution networks such as exhaustive search, ant colony optimization, and cuckoo search algorithm [12-16].

The meta-heuristic is generally known as an approach that cansolve network reconfiguration problems. Several meta-heuristic algorithms have been proposed in the literature for solving these problems $[17,18]$. Comparative studies on various optimization methods show that thegenetic algorithm (GA)and particle swarm optimization (PSO)approaches are the most widely used methods for solving combinatorial problems [19-21]. One of the advantages of these algorithms is that the solution of a problem can still be obtained even there is no analytical solution for the problem [22]. Therefore, the emphasis will be placed on the use of GA and PSO algorithms to solve the problem of finding an optimal configuration of a distribution network.

In a distribution network reconfiguration problem, aload flow algorithm is a fundamental tool for analysis, optimization, and planning. It is an important step for each generation of optimization algorithms. In this paper, the new development introduced in the GA and PSO application to the distribution network reconfiguration problem is the proposed load flow algorithm.

Several load flow algorithms specificallydesigned for distribution systems have been proposed in the literature [23]. From those methods, the Gauss implicit matrix method is one of the most commonly used methods; however, this method does not explicitly exploit 
the radial and weakly meshed network structure of distribution systems. Recent research proposed some new ideas on how to deal with the special topological characteristics of distribution systems [24], but these ideas require new data formats or some data manipulations. To cope with this problem, in this study, a new load-flow method was proposed and introduced in the GA and PSO algorithms for solving the distribution network reconfiguration problem in order to enhance reliability and reduce power losses. The only input data of this algorithm is the conventional bus-branch oriented data used by most utilities. The novelty in this study is to develop a formulation, which takes advantage of the topological characteristics of distribution systems and solves the distribution load flow directly. It means that the time-consuming forward/backward substitution of the Jacobian matrix or the admittance matrix, required in the traditional Newton Raphson and Gauss implicit matrix algorithms, are not necessary in the new development. Two developed matrices, the bus-injection to branch-current matrix and the branch-current to bus-voltage matrix, and a simple matrix multiplication are utilized to obtain load flow solutions.

This paper is organized as follows: Section 2 presents a mathematical simulation of the distribution network reconfiguration problem, as well as all the constraints that must be met and the methods for solving the optimization problem. Section 3 discusses the application of these optimization tools ina distribution network. Section 4 finally draws conclusions on the results of the study.

\section{Materials and Methods}

This section described the tools and methodology that were used to achieve the results presented in this work.

\subsection{Mathematical Formulation of the Distribution Network Reconfiguration Problem}

\subsubsection{Generalities}

We first define a criterion optimization problem, also known as an objective function, as part of the research study. The latter can be subject to a set of constraints. A monoobjective optimization problem is commonly expressed mathematically as follows [22]:

$$
\begin{gathered}
\text { Optimize }[F(x)] \\
\text { Under the constraints : }\left\{\begin{array}{c}
g(x)=0 \\
h(x) \leq 0 \\
x_{i}^{\min } \leq x \leq x_{i}^{\max } \\
x=\left\{x_{1}, x_{2}, \ldots, x_{n}\right\}
\end{array}\right.
\end{gathered}
$$

where $F(x)$ is the mono-objective function to be optimized, $x$ is the vector of $n$ variables representing the parameters of the problem to be optimized, $g(x)$ and $h(x)$ are, respectively, the set of constraints of equality and inequality, and $x_{i}^{\min }$ and $x_{i}^{\max }$ are the domain of constraints.

\subsubsection{Formulation of the Objectives Function for the Reconfiguration Problem}

The goal of this study is to find the best distribution network reconfiguration that reduces both the ENS index and the active power losses while maintaining a set of constraints. The objectives of the optimization problem are detailed as follows.

\section{- Energy not supplied criterion}

The reliability of distribution networks has become increasingly important in the deregulated power sector. To address these issues, distribution network reconfiguration is regarded as a cost-effective and efficient way to increase system reliability. The ENS index is used in distribution system planning studies to refer to customer interrupted electricity. The majority of customer interruptions are caused by distribution system component failures. The ENS index forecasts the amount of energy that will be lost due to failures over a given time period. Based on the accurate estimation of the ENS index, appropriate planning and design initiatives in distribution systems can be implemented to provide consumers with 
cost-effective and reliable power. The ENS is one of the most significant reliability indices that must be included in the distribution network reconfiguration formulation in order to enhance reliability. This study aims to find a radial operating structure that minimizes the ENS while satisfying operational constraints in order to increase customer reliability worth.

We used Equation (2) [25] to calculate the total reliability index ENS, which assesses the quality of supply of all customers.

$$
f_{\text {objectif } 1}=E N S=\sum_{j=2}^{N_{c}} E N S_{j}=\sum_{j=2}^{N_{c}} P_{j} \times \sum_{\substack{i=1 \\ i \neq j}}^{n_{b}} \lambda_{i j} \times l_{i j} \times t_{i j}
$$

where

$P_{j}$ is the active power of the unpowered load point $j[\mathrm{~kW}]$, and $n_{b}$ is the number of radial configuration branches.

$N_{c}$ is the total number of consumers.

$\lambda_{i j}, l_{i j}$, and $t_{i j}$ are, respectively, the failure rate [fault $/ \mathrm{km}$. year], length [km], and the repair and commissioning time of the line $(i, j)[\mathrm{h} /$ fault $]$.

The minimization of the ENS criterion contributes to the maximization of the reliability of various configurations. Therefore, the configuration with the lowest index is the most reliable.

- Active power losses criterion

The distribution networks constitute the majority of the electric power system. This results in a large amount of active power loss. To this end, this criterion cannot be ignored in the distribution network planning problem. Reducing this objective culminates in an optimal distribution network reconfiguration. The mathematical formulation of this problem can be represented as [22]

$$
f_{\text {objectif } 2}=\sum_{i=1}^{N} \sum_{j=i+1}^{N} R_{i j} \times I_{i j}{ }^{2}
$$

where $N$ is the total number of nodes, $R_{i j}$ is the resistance of the line $(i, j)$, and $I_{i j}$ is the electrical current flowing through the line $(i, j)$.

It was important to determine the voltage magnitude and phase angle of each node in order to obtain an accurate expression for the system power losses. Therefore, a load-flow calculation was required. In this context, a new load-flow method for radial distribution networks is proposed.

\subsubsection{Formulation of Constraints}

The various constraints related to the voltages of the network nodes and to the transits of the currents in the lines are called security constraints.

\section{- Voltage constraints}

The voltage constraint is expressed in an optimization algorithm in the following manner [25]:

$$
V_{i}^{\min } \leq V_{i} \leq V_{i}^{\max }
$$

where

$V_{i}$ is the voltage at node ' $i$ ' of the network.

$V_{i}^{\text {min }}$ : minimum voltage, $-10 \%$ of nominal voltage ( $\left.1 \mathrm{pu}\right)$.

$V_{i}^{\max }$ : maximum voltage, $+10 \%$ of nominal voltage $(1 \mathrm{pu})$. 


\section{- Currents constraints}

In addition to the voltage constraints, the currents transmitted through the branches require special attention from distribution network operators. These currents must not exceed the steady-state thresholds. This constraint can be expressed mathematically (5) [25]:

$$
\mathrm{I}_{\mathrm{ij}} \leq \mathrm{I}_{\mathrm{ij}}^{\max \_a d m}
$$

where $I_{i j}$ represents the electrical current transited in a branch $(i, j)$, and $I_{i j}^{\text {max } \_a d m ~ r e p r e s e n t s ~}$ the maximum current in the branch $(i, j)$. These currents were calculated for each topology using the load-flow program [25].

- Topological constraints

When looking for a topology that meets the aforementioned criteria, a set of topology constraints must be observed in addition to security constraints. The desired radial topology must be equivalent to a spanning tree that models the general structure of a network when topology constraints are translated into a graph theory. To ensure compliance with these constraints, it is mandatory that this spanning tree must adhere to the following:

- Contain $(N-1)$ edges, where $N$ is the total number of vertices in the graph;

- Include all the graph's vertices (we must then ensure that the graph is connected);

- Be characterized by the absence of loops

The topological constraints are given by (6) and (7)

$$
\begin{gathered}
N b_{\text {closed lines }}=N-1 \\
\forall x_{i}, x_{j} \in N, \exists\left\{C_{i} \cup C_{j}\right\}, \text { let } \prod_{k}^{n} Z i_{k} \cdot \prod_{k}^{n} Z j_{k}=1
\end{gathered}
$$

$\mathrm{Nb}$ closed lines: number of closed network branches.

$N$ : total number of nodes.

$C_{i} \cup C_{j}$ : unique path connecting node $\mathrm{i}$ and node $\mathrm{j}$.

$Z i_{k,}, Z j_{k}$ : topological states $(0 / 1)$ of the $n$ branches that constitute the path $C_{i} \cup C_{j}$.

$k$ : index of the branches that constitute the path.

\subsection{Proposed Load-Flow Approach}

In this study, the proposed load flow algorithm uses the distribution network tree to determine the direction of the currents flowing through different branches and then calculates their values as well as the various node voltage profiles. The load flow algorithm is based on two matrices derived from the network topology: the bus injection branch current (BIBC) matrix and the branch current bus voltage (BCBV) matrix. A flowchart of the proposed load flow is shown in Figure 1. For the proposed load-flow method, each node is supplied by a unique source node; however, a source node can supply more than one bus. Initially, the node voltages were considered equal to the unity, with the exception of the source bus voltage, which was assumed to be equal to its nominal value. Moreover, it was assumed that the shunt capacitances were negligible.

\subsection{Methods for Solving the Optimization Problem}

In this study, three methods are used to solve the distribution network reconfiguration problem: the exhaustive approach, GA approach, and PSO approach. 


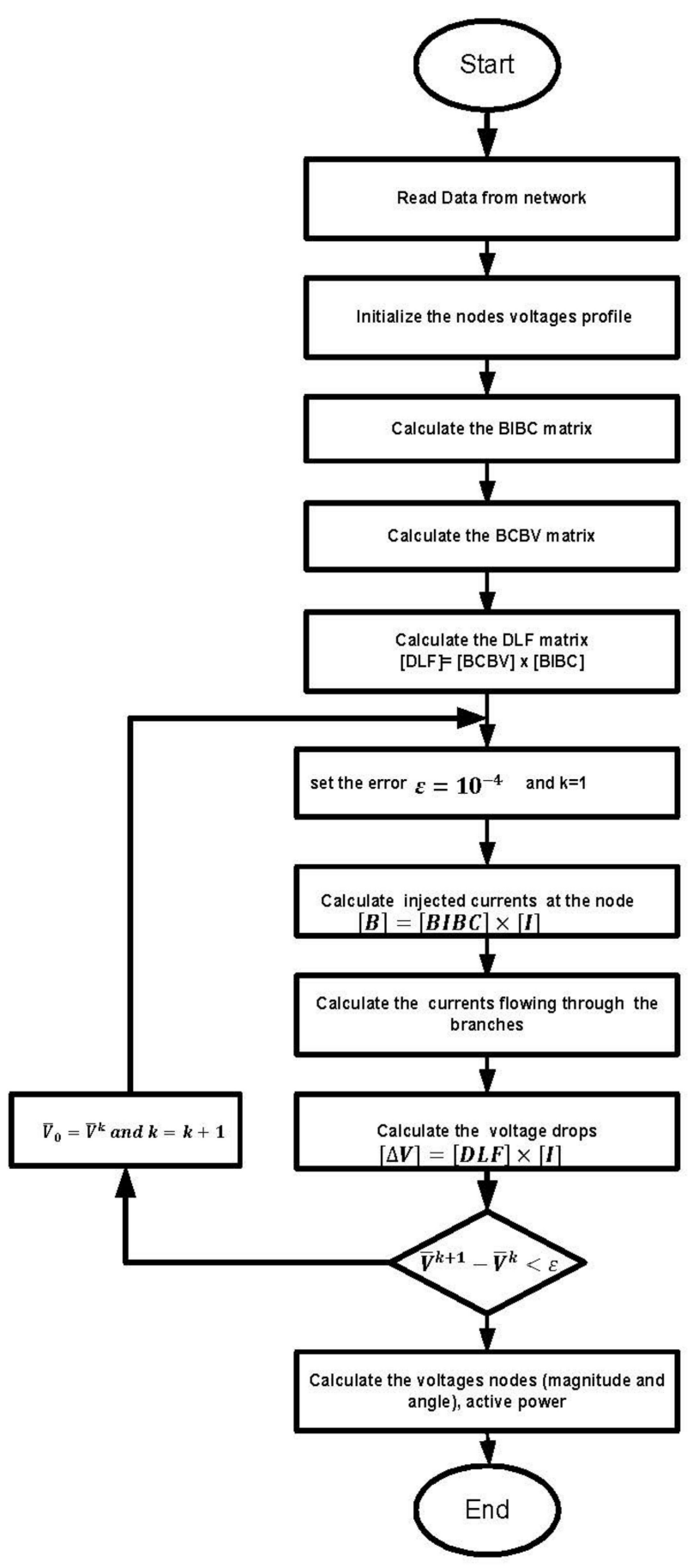

Figure 1. Flowchart of the proposed load flow.

\subsubsection{Exhaustive Approach}

The exhaustive approach entails explicitly enumerating all possible configurations, evaluating them, and finally identifying the one that best satisfies the criterion to be minimized [17]. In this study, we considered this exhaustive approach in order to compare the different results obtained by other solving techniques.

To find the optimal configuration, the set of solutions can be determined by considering the two possible states of each maneuverable line. As mentioned earlier, the total number of solutions is $2^{M}$, where $M$ represents the number of maneuverable branches. We then calculated the combinations of the total number of operable lines and the total 
number of closed branches. The number of solutions given by the combinatorial formula (5) represents the maximum number of solutions that a search algorithm can explore.

$$
N b_{\text {sol }} l_{\text {possibles }}=\complement_{M}^{M-N}
$$

where $M$ is the number of branches that can be operated and $N$ is the number of branches to be opened.

The flowchart shown in Figure 2 provides a basic illustration of the exhaustive approach.

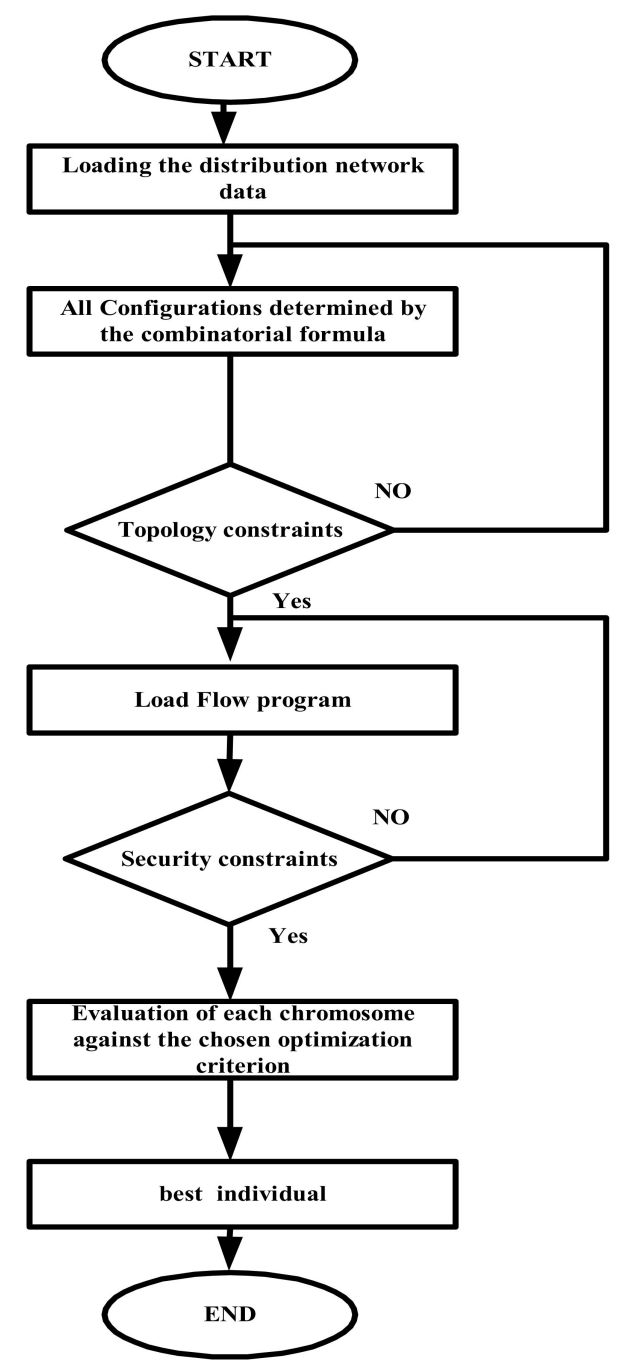

Figure 2. Flowchart of the exhaustive approach.

\subsubsection{GA Approach}

The developed optimization algorithm for distribution network reconfiguration in this section is based on the GA method. The basic principle of GA is to evolve a population of individuals using genetic operators (selection, crossover, and mutation) [26]. During each generation procedure, these operators are applied to each person to generate a new evolved population from the previous one. In GA, each person is represented by a single chromosome. According to graph theory, this chromosome corresponds to a radial distribution network configuration or a spanning tree of a given graph. The group of chromosomes is referred to as the population. In GA implementation, an initial population of individuals is randomly generated as a first step. Then, each chromosome is encoded. In this study, the network topology is represented by only considering closed branches. A real coding strategy, in which each gene corresponds to an edge of the spanning tree, was used. Fol- 
lowing that, each person was evaluated. Three genetic operations are used to accomplish the proposed GA: crossover, mutation, and selection. A total of two crossover points were considered. These points were randomly selected and they split the chromosome into three segments. The second segments from two identified chromosomes were switched in the crossover operation. In this study, the crossover rate is 0.9 . The mutation rate is set to be 0.01 to prevent a local optimum. The tournament approach is used for selecting the individuals. The flowchart of the GA approach is presented in Figure 3.

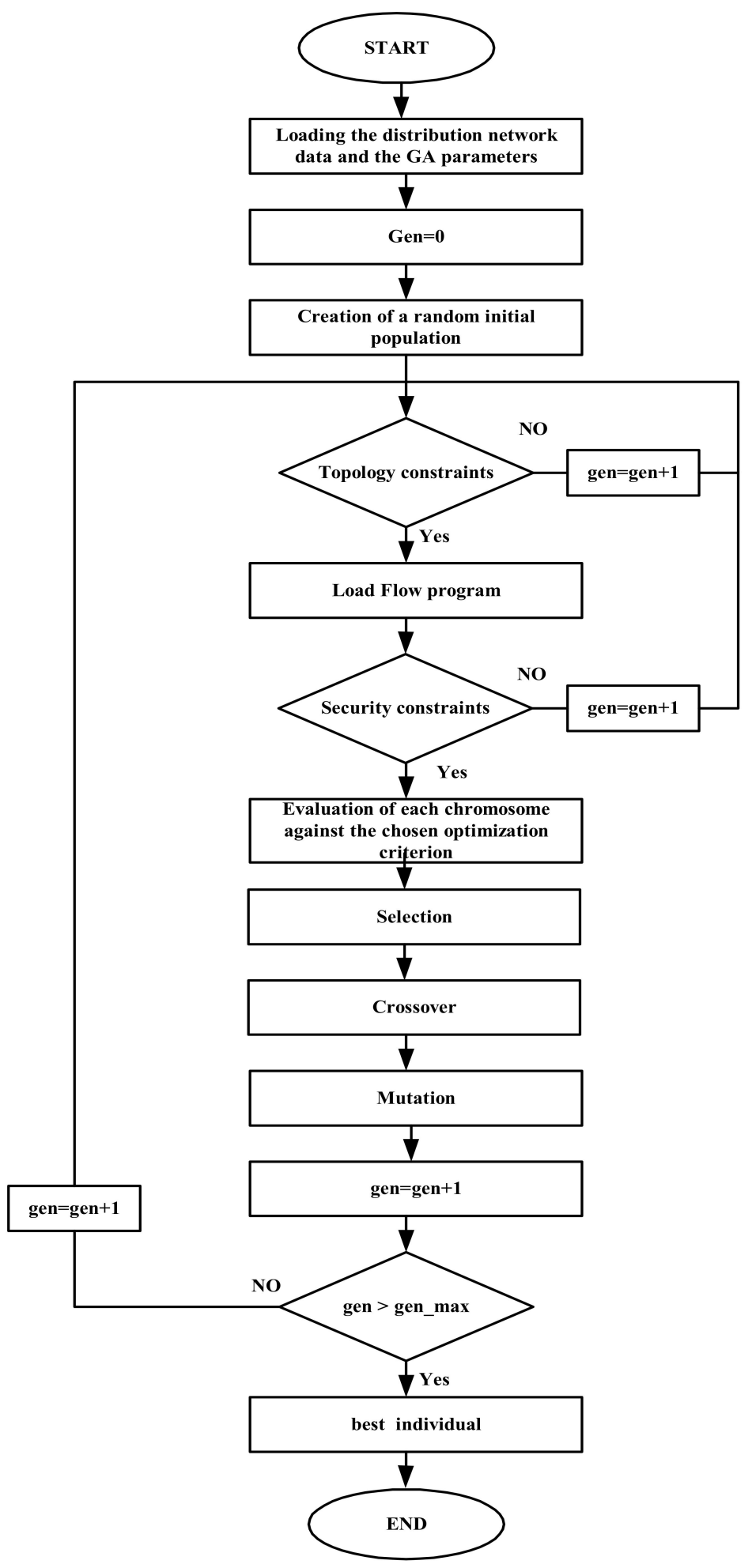

Figure 3. Flowchart of the genetic algorithm (GA)approach. 


\subsubsection{PSO Approach}

In PSO, each particle's coordinates represent a possible solution that is associated with two vectors, the position $\left(x_{i}\right)$ and velocity $\left(v_{i}\right)$ vectors $[27,28]$. The size of vectors $x_{i}$ and $v_{i}$ is equal to the number of particles. A swarm consists of a number of particles or "possible solutions" that proceed (fly) through the feasible solution space to explore optimal solutions. Each particle's position is updated based on its own best exploration, overall swarm experience, and previous velocity vector. The update rules with inertial weight for particle $i$ at iteration $\mathrm{k}+1$ are given by (9) and (10).

$$
\begin{gathered}
v_{i}^{k+1}=w v_{i}^{k}+c_{1} r_{1}\left(p_{i}^{k}-x_{i}^{k}\right)+c_{2} r_{2}\left(g_{i}^{k}-x_{i}^{k}\right) \\
x_{i}^{k+1}=x_{i}^{k}+v_{i}^{k+1}
\end{gathered}
$$

where $v_{i}^{k}, x_{i}^{k}$ are the velocity and position of particle $i$, respectively; $w$ is the inertia weight; $c_{1}$ and $c_{2}$ are the acceleration coefficients; $r_{1}$ and $r_{2}$ are two random numbers between 0 and $1 ; p_{i}^{k}$ is the best position found so far for the $i$ th particle, $g_{i}^{k}$ is the best position found so far by the entire swarm. Figure 4 shows the flowchart of the PSO approach.

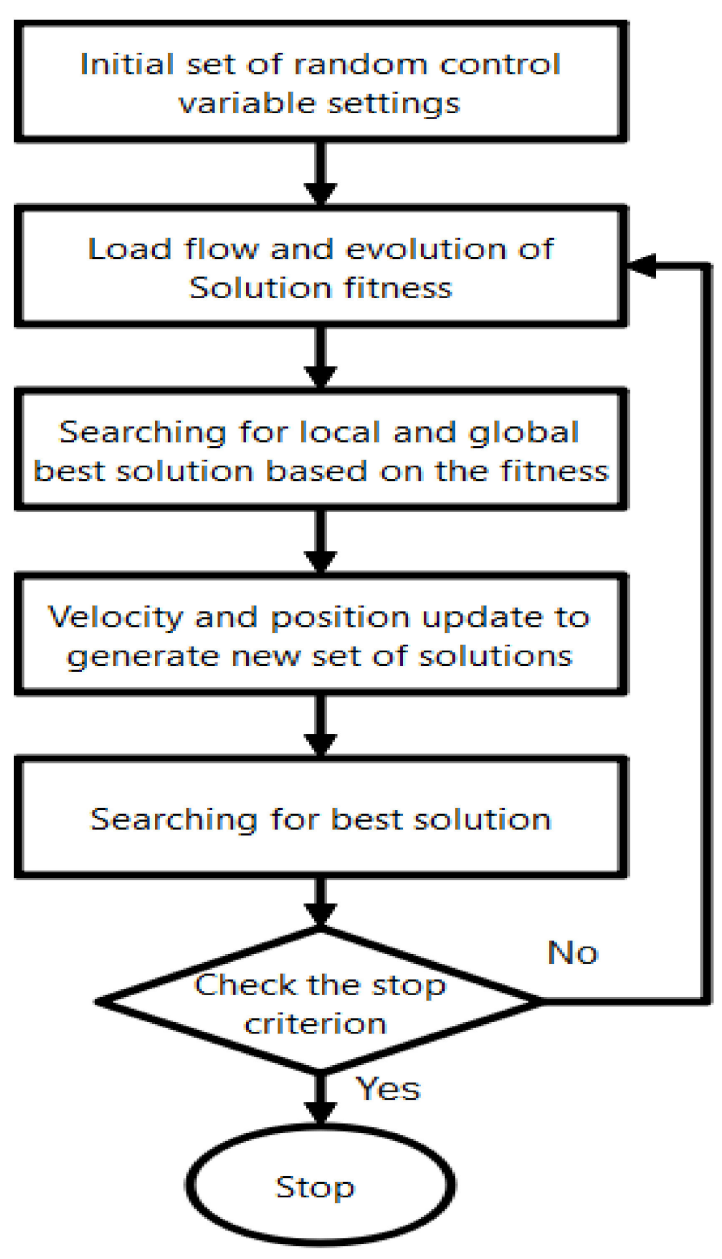

Figure 4. Flowchart of the particle swarm optimization (PSO) approach.

\section{Simulation Results}

The distribution network reconfiguration problem had two objectives: the first was to minimize the ENS, and the second was to optimize the reduction of active power losses. 


\subsection{IEEE 33-Bus Test System}

Figure 5 depicts the IEEE 33-bus distribution network used in this analysis. It is made up of 33 nodes and 37 branches. The distribution network data (line data, bus data) are available in [25]. Initially, the open branches (OB) were as follows [33-34-35-36-37]. The ENS and active power losses for the initial configuration were $7.0980 \mathrm{kWh} /$ year and $202.6774 \mathrm{~kW}$, respectively. The MATLAB environment was used to program the proposed load flow and implement the proposed optimization approaches.

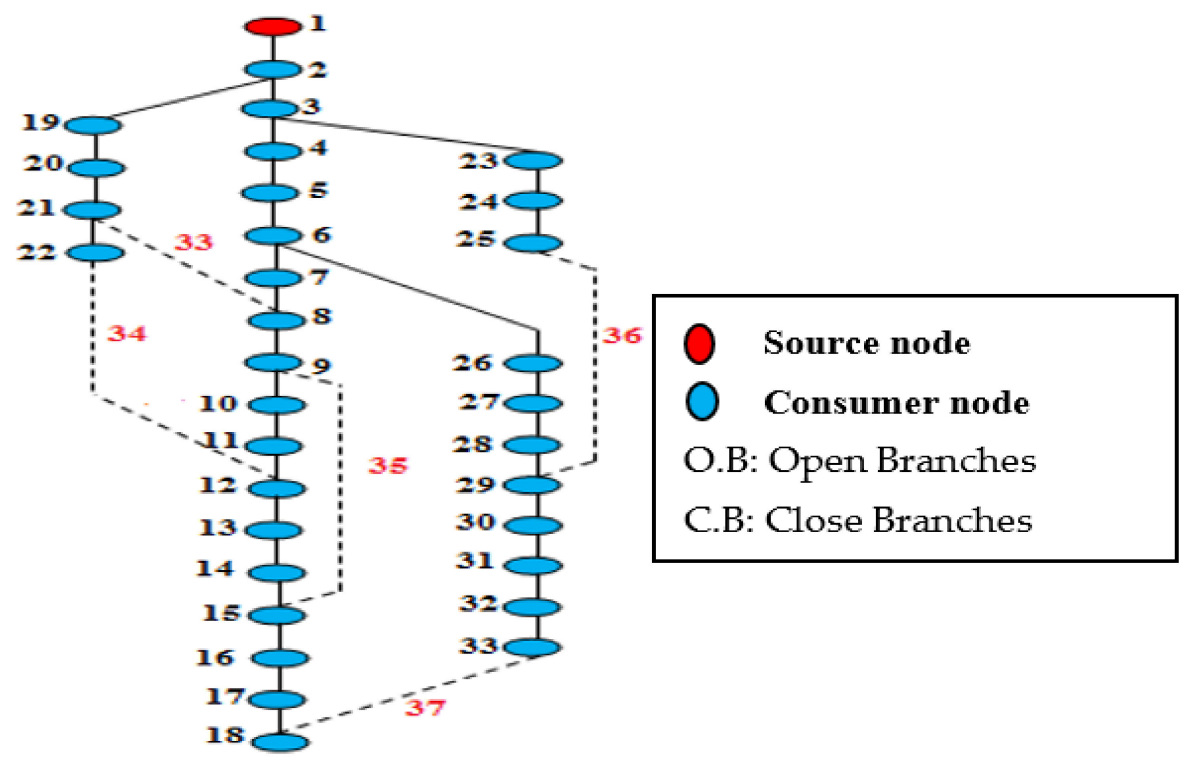

Figure 5. IEEE 33-bus test system.

\subsection{Minimization of the ENS Criterion}

In this section, for the ENS criterion, the optimal topology for the IEEE 33-bus test network is obtained using the exhaustive approach, GA, and PSO algorithms. Based on the combinatorial formula, the number of solutions to explore in this case is equal to $\mathrm{Nb}_{\text {sol }}=435.897$ solutions. There are a total of 50,751 radial configurations for this distribution network. Subsequently, using the proposed load-flow program, each of these configurations must be evaluated against the optimization criterion while adhering to all safety constraints.

Table 1 summarizes the characteristics of the three methods developed to solve the mono-objective optimization problem infindingan optimal configuration for the IEEE 33bus test system. The PSO approach appears to be the most efficient. In comparison to the AG and exhaustive research, it requires less computational effort.

Table 1. Comparison between the proposed approaches for the minimization of the energy not supplied (ENS)criterion.

\begin{tabular}{cccc}
\hline & $\begin{array}{c}\text { Optimal Configuration } \\
\text { (Open Branches) }\end{array}$ & $\begin{array}{c}\text { ENS } \\
\text { (kWh/an) }\end{array}$ & Calculation Time (s) \\
\hline Exhaustive approach & $7-9-14-16-27$ & 4.7304 & $1.296 \times 10^{-4}$ \\
GA & $7-9-14-16-27$ & 4.7304 & 335.14 \\
PSO & $7-9-14-16-27$ & 4.7304 & 150.36 \\
\hline
\end{tabular}

The optimal network configuration obtained for the minimization of the ENS criterion is shown in Figure 6. The open branches in this case are [7-9-14-16-27]. The ENS in this radial structure is $4.7304 \mathrm{kWh} /$ year, which is $66.64 \%$ less than the initial topology (7.0980 kWh/year). 
A good voltage profile with regard to the initial topology was obtained by maximizing the reliability (Figure 7). The voltages are as close to the nominal voltage (1 pu) as possible. As a result, voltage profiles were improved in all network nodes. It is also evident that the security constraints are observed.

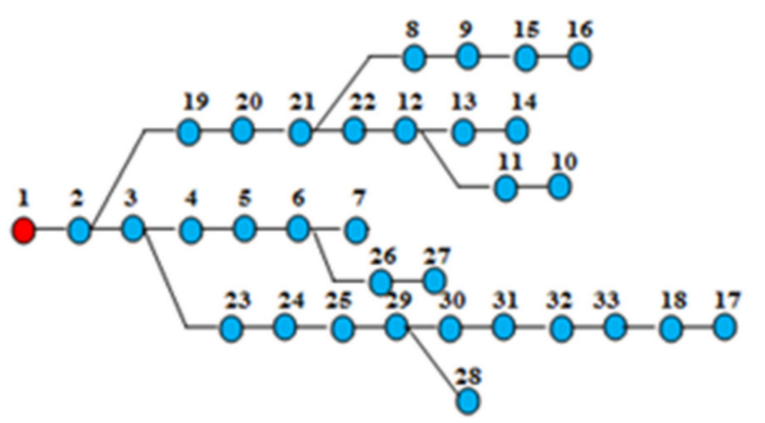

Figure 6. Optimal configuration for the minimization of the ENS criterion (open branches: 7-9-14-16-27).

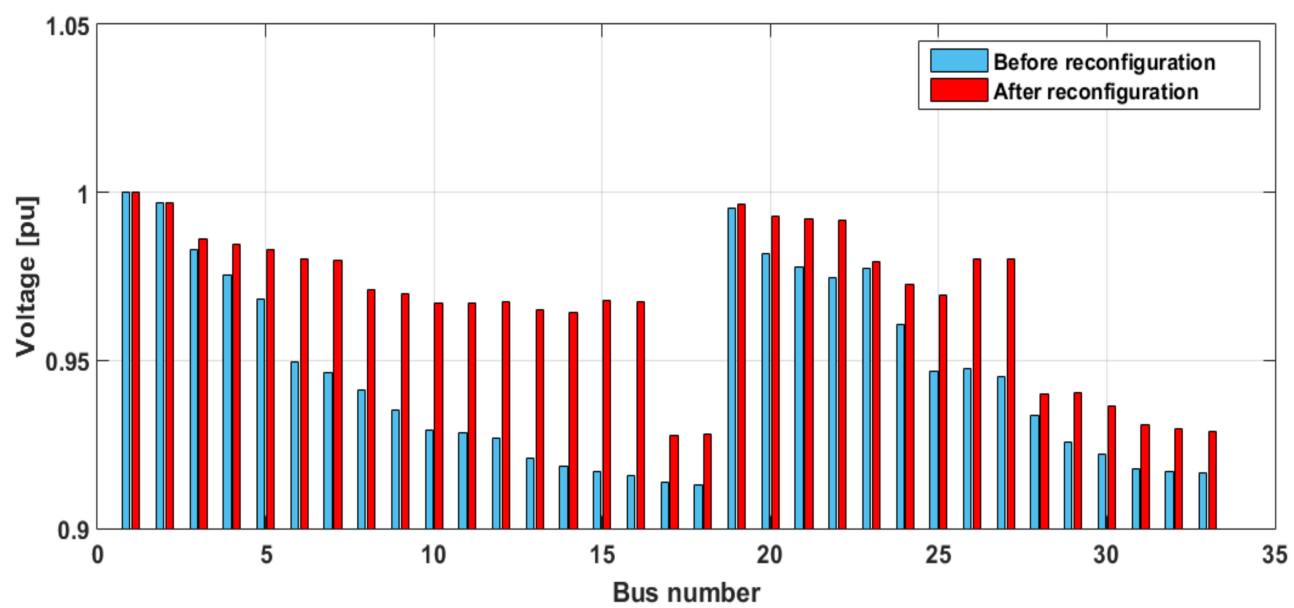

Figure 7. Voltage profiles for the minimization of the ENS criterion (before and after reconfiguration)).

In terms of the transited current restriction, we can observe from Figure 8 that all currents passing through the distribution lines were within their allowable limits. We also observed that the profile of the currents per branch was generally lower after the reconfiguration process.

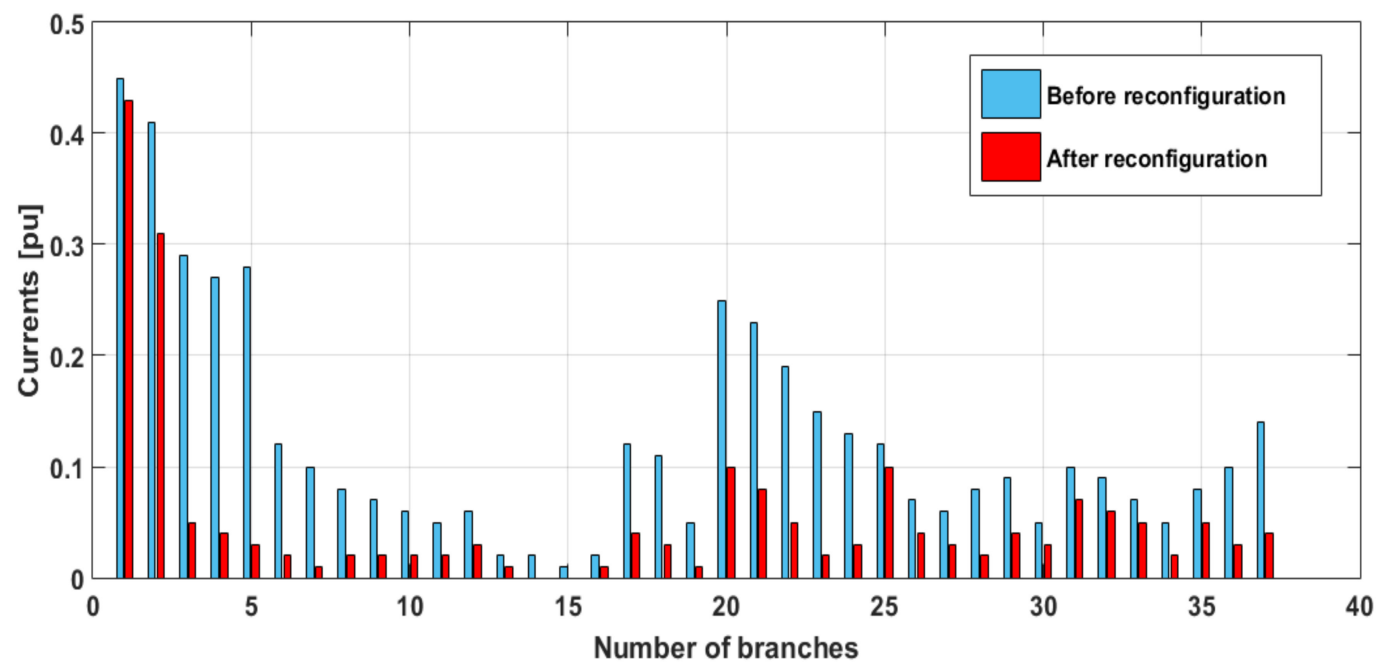

Figure 8. Currents profiles for the minimization of the ENS criterion (before and after reconfiguration)). 
Figure 9 shows the GA and PSO convergence characteristics of ENS for the IEEE 33-bus test system. From this figure, it is evident that the PSO algorithm outperforms the other algorithms in terms of speed to reach the optimal solution. The difference in terms of iterations in achieving an optimal solution was 25 iterations. Both algorithms achieved an optimal solution. However, compared to GA, the PSO approach appears to be more appealing due to its lower computational effort. This is demonstrated by the fact that, unlike the GA, we do not need to replicate the constraint validation test at each iteration. Therefore, this approach is considered one of the fastest techniques for solving distribution network optimization problems.

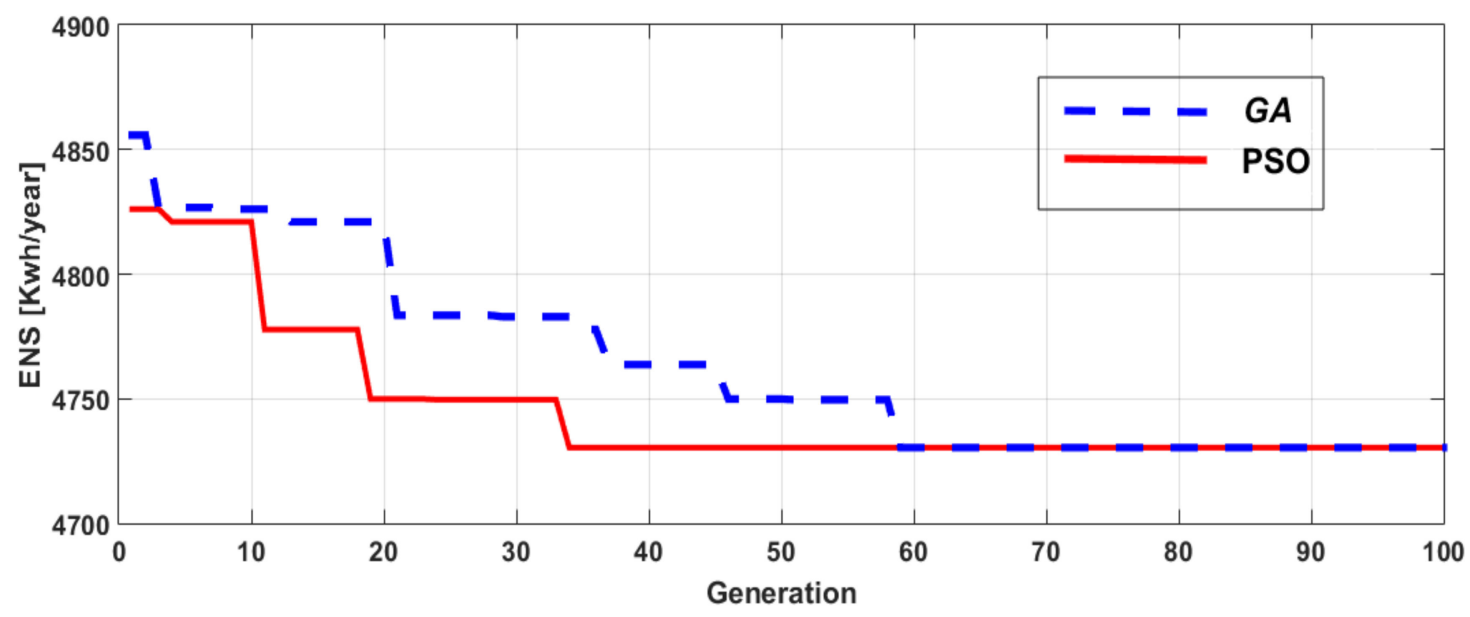

Figure 9. GA and PSO convergence characteristic for the minimization of the ENS criterion.

\subsection{Minimization of the Active Losses Criterion}

Relying on the load-flow method proposed in this study, the calculation of the active power losses in each branch was achieved. In this case, the ultimate aim was to reduce the power losses by applying the proposed approaches.

The results presented in Table 2 show that the active power losses decreased by $63.1258 \mathrm{~kW}$ compared to the base case value of $202.6774 \mathrm{~kW}$. The percentage reduction was $31.15 \%$ compared with the initial topology presented in Figure 5. The open branches are 7-9-14-32-37 as indicated in Figure 10.

Table 2. Comparison between the proposed approaches for the minimization of the active power losses.

\begin{tabular}{ccccc}
\hline & $\begin{array}{c}\text { Optimal Configuration } \\
\text { (Open Branches) }\end{array}$ & Success Rate (\%) & $\begin{array}{c}\text { Active Power Losses } \\
(\mathbf{k W})\end{array}$ & Calculation Time (s) \\
\hline Exhaustive approach & $7-9-14-32-37$ & 82 & 139.5516 & $1.123 \times 10^{+6}$ \\
\hline GA & $7-9-14-32-37$ & 61 & 139.5516 & 328.63 \\
\hline PSO & $7-9-14-32-37$ & 8 & 139.5516 & 120.25 \\
\hline
\end{tabular}

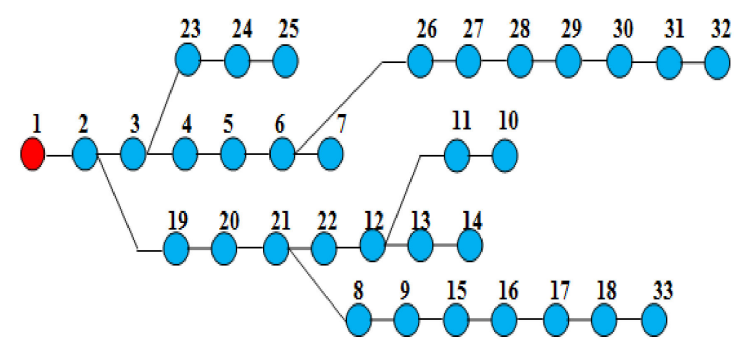

Figure 10. Optimal configuration for the minimization of the active power losses criterion (open branches: 7-9-14-32-37). 
The simulation results show that, for the IEEE 33-bus test system, all methods have found the optimal network configuration with minimum power loss. However, the number of runs reaching out to the optimal configuration of PSO is superior to the remaining methods. Specifically, the success rate, which is defined as a result obtained in percent by dividing the number of runs finding out the optimal configuration by the total of runs, of PSO is $85 \%$ while this value of GA and exhaustive approaches are 61 and $8 \%$. Figure 11 shows the comparisons of the successful rate of the proposed methods for IEEE 33-bus test system.

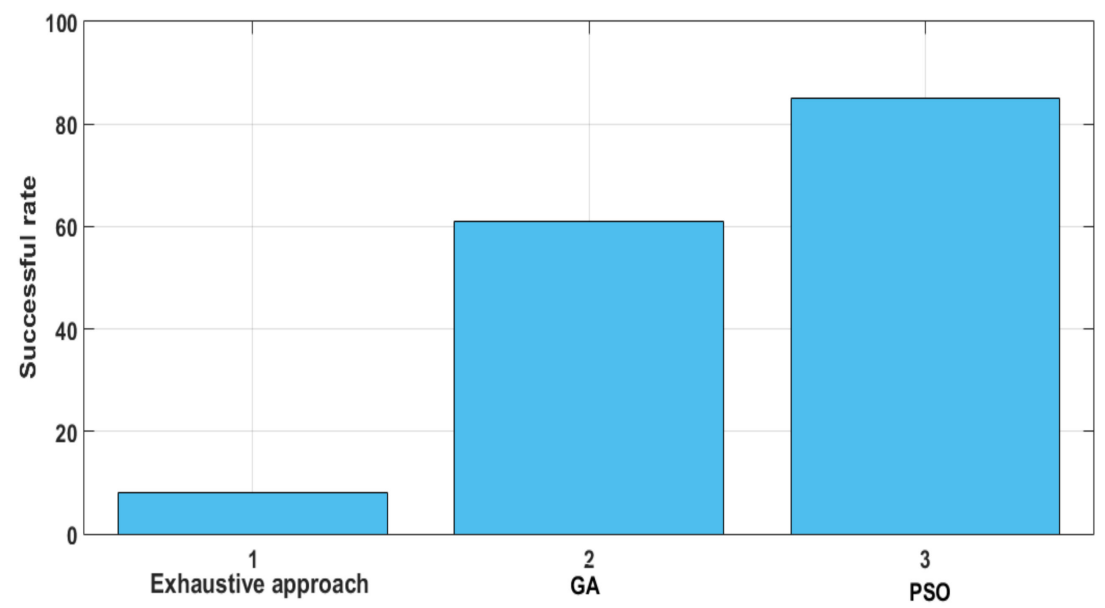

Figure 11. The comparison of the successful rate of the proposed methods for IEEE 33-bus test system.

The evolution of the active power losses during the searching process using the GA and PSO algorithms for the test distribution system is shown in Figure 12. The difference in terms of iterations in achieving an optimal solution was 14 iterations. Although GA is superior to the exhaustive approach, it is still inferior to PSO in terms of speed to reach the optimal solution. In addition, this method can solve, in a satisfactory manner the problem of finding the optimal configuration for a large network in a reasonable time.

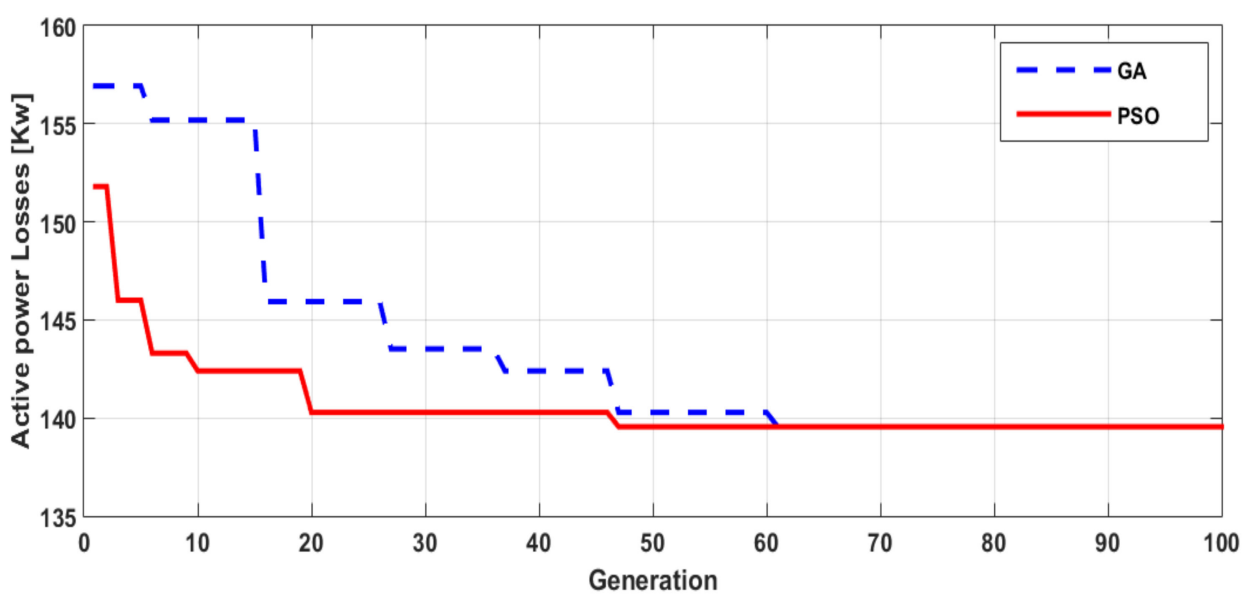

Figure 12. GA and PSO convergence characteristic for the minimization of the active power losses criterion.

The PSO-based reconfiguration solution proposed in this paper provides significant improvements in active power losses and reliability of the IEEE 33-bus distribution network. Indeed, the PSO approach is a population-based evolutionary technique that has many key advantages over other optimization techniques. First, it is a derivative-free algorithm, unlike many conventional techniques. Second, it has the flexibility to be integrated with other techniques to form a hybrid tool such as a load flow algorithm. Then, it 
has fewerparameters to adjust, unlike many other competing evolutionary techniques. It also has the ability to escape from local minima. Finally, it does not require a good initial solution to start its iteration process.

\section{Conclusions}

In this paper, the network reconfiguration problem has been considered for reliability enhancement and active power loss reduction. To enhance the efficiency of the metaheuristic algorithms for the network reconfiguration problem, an effective method is proposed. The idea of this method is to develop a formulation, which takes advantage of the topological characteristics of distribution systems and solves the distribution load flow directly. The objective functions for the network reconfiguration considered in this work are the active power losses and ENS index. The constraints considered in this study are security and topology constraints. Optimizing the considered objective functions allows us to deal with mono-objective optimization problems. In this context, exhaustive, GA, and PSO approaches to distribution reconfiguration are proposed. The results show that PSO is superior to exhaustive and GA approaches in terms of the success rate for finding the optimal network configuration and the number of convergence iterations. The voltage and current profiles obtained from simulation results show that all the node voltage amplitudes and the current of branches have been changed more positively than before reconfiguration. The result comparison indicated that the proposedPSO approach provides faster convergence than the exhaustive approach and GA, as shown by the convergence graph in Figures 9 and 12. The performance of the proposed PSO approach is better and more appropriate for practical applications in power system reconfiguration.

Author Contributions: Conceptualization, H.A.; methodology, Y.B.; software, Y.B.; validation, N.B.A. and O.K.; formal analysis, Y.B.; investigation, H.A.; resources, M.C.; data curation, M.C.; writingoriginal draft preparation, O.K.; writing-review and editing, O.K.; visualization, H.A.; supervision, M.C.; project administration, N.B.A.; funding acquisition, N.B.A. All authors have read and agreed to the published version of the manuscript.

Funding: This research has been funded by Scientific Research Deanship at University of Ha'il Saudi Arabia through project number RG-20 021.

Institutional Review Board Statement: Not applicable.

Informed Consent Statement: Not applicable.

Acknowledgments: This research has been funded by Scientific Research Deanship at University of Ha'il-Saudi Arabia through project number RG-20 021.

Conflicts of Interest: The authors declare no conflict of interest.

\section{References}

1. Rojas, A.L.; Koziel, S.; Abdel-Fattah, M.F.; Gutiérrez-Alcaraz, G. Distribution Network Reconfiguration for Voltage Stability Enhancement via Feasibility-Preserving Evolutionary Optimization. In Proceedings of the Electrical Power and Energy Conference (EPEC) 2018 IEEE, Toronto, ON, Canada, 10-11 October 2018; pp. 1-8.

2. Ma, Y. The review on distribution network reconfiguration. In Proceedings of the 201729 Chinese Control and Decision Conference (CCDC), Chongqing, China, 28-30 May 2017. [CrossRef]

3. Oloulade, A.; Imano, A.; Fifatin, X.; Vianou, A.; Tamadaho, H.; Badarou, R. Multi-Objective Optimization of the Safe Operation of the Electrical Distribution System by Placing D-FACTS and Network Reconfiguration. J. Power Energy Eng. 2019, 7, 94-113. [CrossRef]

4. Farid, A.K.; Nikna, T. Multi-objective stochastic distribution feeder reconfiguration from the reliability point of view. Energy 2014, 64, 342-354. [CrossRef]

5. The, T.T.; Ngoc, D.V.; Anh, N.T. Distribution Network Reconfiguration for Power Loss Reduction and Voltage Profile Improvement Using Chaotic Stochastic Fractal Search Algorithm. Complexity 2020, 2020, 2353901. [CrossRef]

6. Sultana, B.; Mustafa, M.W.; Sultana, U.; Bhatti, A.R. Review on reliability improvement and power loss reduction in distribution system via network reconfiguration. Renew. Sustain. Energy Rev. 2016, 66, 297-310. [CrossRef]

7. Mendoza, J.; López, M.E.; Coello, C.A.C.; López, E.A. Microgenetic Multiobjective Reconfiguration Algorithm Considering Power Losses and Reliability Indices for Medium Voltage Distribution Network. IET Gener. Trans. Distrib. 2009, 3, 825-840. [CrossRef] 
8. Narimani, M.R.; Vahed, A.A.; Azizipanah-Abarghooee, R.; Javidsharifi, M. Enhanced gravitational search algorithm for multiobjective distribution feeder reconfiguration considering reliability, loss and operational cost. IET Gener. Trans. Distrib. 2014, 8 , 55-69. [CrossRef]

9. Agrawal, P.; Kanwar, N.; Gupta, N.; Niazi, K.R.; Swarnkar, A.; Meena, N.K.; Yang, J. Reliability and Network Performance Enhancement by Reconfiguring Underground Distribution Systems. Energies 2020, 13, 4719. [CrossRef]

10. Amanulla, B.; Chakrabarti, S.; Singh, S.N. Reconfiguration of power distribution systems considering reliability and power loss. IEEE Trans. Power Deliv. 2012, 27, 918-926. [CrossRef]

11. Merlin, A.; Back, H. Search for a minimal-loss operating spanning tree configuration in an urban power distribution system. In Proceedings of the 5th Power System Computation Conference, Cambridge, UK, 1-5 September 1975.

12. Taylor, J.A.; Hover, F.S. Convex models of distribution system reconfiguration. Power Syst. IEEE Trans. 2012, 27, 1407-1413. [CrossRef]

13. Daniel, L.C.; Khan, I.H.; Ravichandran, S. Distribution network reconfiguration for loss reduction using ant colony system algorithm. In Proceedings of the 2005 Annual IEEE India Conference-Indicon, Chennai, India, 11-13 December 2005; pp. 619622.

14. Nguyen, T.T.; Truong, A.V. Distribution network reconfiguration for power loss minimization and voltage profile improvement using cuckoo search algorithm. Int. J. Electr. Power Energy Syst. 2015, 68, 233-242. [CrossRef]

15. Imran, A.M.; Kowsalya, M. A new power system reconfiguration scheme for power loss minimization and voltage profile enhancement using fireworks algorithm. Int. J. Electr. Power Energy Syst. 2014, 62, 312-322. [CrossRef]

16. Su, C.; Chang, C.; Chiou, J. Distribution network reconfiguration for loss reduction by ant colony search algorithm. Elect. Power Sys. Res. 2005, 75, 190-199. [CrossRef]

17. Sedghi, M.; Ahmadian, A.; Aliakbar-Golkar, M. Assessment of optimization algorithms capability in distribution network planning: Review, comparaison and modification techniques. Renew. Sustain. Energy Rev. 2016, 415-434. [CrossRef]

18. Baghel, M.; Agrawal, S.; Silakari, S. Survey of Metaheuristic Algorithms for Combinatorial Optimi-zation. Int. J. Comput. Appl. 2012, 58. [CrossRef]

19. Ramesh, L.; Chowdhury, S.; Chowdhury, S.; Natarajan, A.; Gaunt, C. Minimization of power loss in distribution networks by different techniques. Int. J. Electr. Power Energy Syst. Eng. 2009, 2, 1-6.

20. Davalos, F.; Irving, M.R. An efficient genetic algorithm for optimal largescale power distribution network planning. In Proceedings of the 2003 IEEE Bologna Power Tech Conference Proceedings, Bologna, Italy, 23-26 June 2003.

21. Duran, M.Q.; Candelo, J.E.; Sousa, V. Recent Trends of the Most Used Metaheuristic Techniques for Distribution Network Reconfig-uration. J. Eng. Sci. Technol. Rev. 2017, 10, 159-173. [CrossRef]

22. Souifi, H.; Kahouli, O.; Abdallah, H.H. Multi-objective Distribution Network Reconfiguration Op-timization Problem. Electr. Eng. J. 2019, 101, 45-55. [CrossRef]

23. Singh, S.; Ghose, T. Improved radial load flow method. Int. J. Electr. Power Energy Syst. 2013, 44, 721-727. [CrossRef]

24. Murty, V.V.S.N.; Teja, B.R.; Kumar, A. A contribution to Load Flow in Radial Distribution System and Comparison of Different Load FlowMethods. In Proceedings of the International Conference on Power Signals Control and Computations (EPSCICON), Thrissur, India, 6-11 January 2014.

25. Kahouli, O. Contribution to the Improvement of Control Strategies and Electrical Network Planning; HDR, University of Sfax: Sfax, Tunisia, 2019.

26. Jebali, M.; Kahouli, O.; Abdallah, H.H. Optimizing PSS parameters for a multi-machine power system using genetic algorithm and neural network techniques. Int. J. Adv. Manuf. Technol. 2017, 90, 2669-2688. [CrossRef]

27. Dong, W.; Zhou, M. A Supervised Learning and Control Method to Improve Particle Swarm Optimization Algorithms. In IEEE Transactions on Systems, Man, and Cybernetics Systems; IEEE: Piscataway, NJ, USA, 2016; pp. 1-14. [CrossRef]

28. Cao, Y.; Zhang, H.; Li, W.; Zhou, M.; Zhang, Y.; Chaovalitwongse, W.A. Comprehensive Learning Particle Swarm Optimization Algorithm with Local Search for Multimodal Functions. IEEE Trans. Evol. Comput. 2019, 23, 718-731. [CrossRef] 